\title{
Componentes de variância genética e ambiental nos padrões de atividade física de gêmeos. Exploração das possibilidades da entropia aproximada
}

Genetic and environmental variance components for physical activity patterns of twins. Exploring the possibilities of approximate entropy

\author{
Ramon de Brito Costa Pinheiro Lima 1 \\ Rogério César Fermino 2,3,4 \\ André Seabra \\ Rui Garganta \\ José António Ribeiro Maia
}

1 Universidade do Porto. Desporto, Faculdade de Desporto. Laboratório de Cineantropometria e Estatística Aplicada. Porto. Portugal.

2 Universidade Federal do Paraná. Programa de Pós-Graduação em Educação Física. Curitiba, PR. Brasil.

3 Pontifícia Universidade Católica de Curitiba. Grupo de Pesquisa em Atividade Física e Qualidade de Vida. Curitiba, PR. Brasil

\section{Bolsista CAPES}

Recebido em 28/09/09 Revisado em 15/12/09 Aprovado em 8/01/10
Resumo - O principal objetivo deste estudo foi estimar a contribuição dos fatores genéticos no padrão da atividade física (AF) de gêmeos através da estatística entropia aproximada (EA). A amostra foi constituída por 162 pares de gêmeos monozigóticos (MZ) e dizigóticos (DZ) com idade entre seis e 18 anos, residentes em Portugal. A AF foi medida pelo acelerômetro Tritrac-R3D durante cinco dias de uma semana habitual. Para descrever o padrão da AF, recorreu-se a descrição da EA estimada através do software Cine Wizard. A determinação da zigotia foi realizada com base no método direto de análise do DNA. Para a análise dos dados, foram utilizados diferentes softwares como SYSTAT 10.0, STATA 10,0 e o Twinan92. A EA foi calculada para três diferentes fenótipos de AF, classificados de acordo com a quantidade de dias de mensuração (EA 5, 3 e 2 dias). Foi utilizado o recurso a modelos distintos para descrever o padrão das matrizes de covariância entre pares de gêmeos (variância genética, envolvimento comum e único) e o nível de significância foi fixado em 5\%. Foi verificada a presença de agregação fraterna na EA com uma maior homogeneidade entre os gêmeos MZ. Pode-se concluir que os fatores genéticos apresentaram forte influência, na variação interindividual, no padrão de AF e sua contribuição variou entre 44 e $89 \%$.

Palavras-chave: Padrão de atividade física; Entropia aproximada; Gêmeos; Fatores genéticos.

Abstract - The main objective of this study was to estimate the contribution of genetic factors to physical activity patterns (PAPs) in twins using approximate entropy (ApEn) statistics. The sample consisted of 162 monozygotic and dizygotic twins from Portugal aged 6 to 18 years. Physical activity was measured with a Tritrac-RT3 triaxial accelerometer over 5 days of a usual week. PAPs were described by ApEn using the Cine Wizard software. Zygosity was assessed by direct DNA analysis. Data were analyzed using the SYSTAT 10, STATA 10 and Twinan92 softwares. PAPs were estimated for 5, 3 and 2 days. In addition, structural equation modeling was used to compute different sources of variance genetic, common environmental and unique environmental variance. The level of significance was set at $5 \%$. Sibling aggregation was identified by ApEn analysis, with monozygotic twins showing greater homogeneity. In conclusion, genetic factors accounted for 44 to $89 \%$ of the total variation in PAP.

Key words: Physical activity pattern; Approximate entropy; Twins; Genetic factors. 


\section{INTRODUÇÃO}

É inegável atribuir os benefícios à saúde de um estilo de vida ativo com níveis moderados a elevados de atividade física (AF), sendo considerada, atualmente, de um ponto de vista metafórico, um "medicamento" eficaz para a redução do risco de doenças como aterosclerose, hipertensão, distúrbios emocionais, obesidade e osteoporose $\mathrm{e}^{1,2}$. Assim, a prática de AF é, atualmente, recomendada por órgãos como o Center of Disease and Control (CDC) dos Estados Unidos, American College of Sports Medicine (ACSM) e World Health Organization $^{1,3}$.

Face à circunstância da AF ser um fenótipo complexo de natureza multifatorial, não devemos atribuir grande parte da variabilidade dos seus valores populacionais a um único agente. Diversos estudos têm verificado que a heterogeneidade na $\mathrm{AF}$, em todas as suas dimensões, associa-se a um conjunto de fatores designados de biológicos, psicológicos, demográficos, comportamentais, sociais/culturais e ambientais ${ }^{4-6}$. Outros estudos têm salientado o forte impacto da genética nos níveis de AF, sendo a sua relevância estimada entre $30 \mathrm{e}$ $83 \%$ da variação total ${ }^{7,8}$.

Um grande estudo realizado em sete países europeus, com uma amostra de 37.051 pares de gêmeos (GenomEUtwin Project), revelou uma consistente influência da genética sobre a AF, com estimativas de heritabilidade que variaram entre $48-71 \%{ }^{9}$. Beunen e Thomis $^{10}$ realizaram um estudo com 91 pares de gêmeos, de 15 anos de idade e verificaram que para os indivíduos do sexo masculino, a magnitude da componente de variância genética $\left(\mathrm{a}^{2}\right)$ foi estimada em $83 \%$, a componente do envolvimento comum $\left(\mathrm{c}^{2}\right) \mathrm{em}$ $0 \%$ e a componente do envolvimento único $\left(\mathrm{e}^{2}\right)$ em $17 \%$. Esses resultados evidenciaram uma forte presença dos fatores genéticos. Entretanto, para o sexo feminino, os resultados foram distintos, onde $\mathrm{a}^{2}=44 \%, \mathrm{c}^{2}=54 \%$ e $\mathrm{e}^{2}=2 \%$, mostrando uma forte influência dos fatores do envolvimento comummente partilhado. Maia et al..$^{11}$ avaliaram 96 pares de gêmeos, de seis à 12 anos de idade e encontraram que cerca de $25 \%$ das diferenças interindividuais nos níveis de AF podem ser atribuídas aos fatores genéticos. Carlsson et al. ${ }^{12}$ verificaram que as correlações de AF eram duas vezes mais elevadas em gêmeos monozigóticos (MZ), quando comparados aos dizigóticos (DZ), sugerindo, dessa forma, a presença de efeitos genéticos. A variação na $\mathrm{AF}$ devido a fatores hereditários foi estimada em 57 e
50\% para homens e mulheres, respectivamente. A influência do ambiente comum na AF apresentouse muito pequena em comparação à influência do ambiente único ${ }^{12}$.

Não obstante a relevância interpretativa das informações supracitadas, não são conhecidos estudos sistemáticos que procuraram interpretar, do ponto de vista da sua variabilidade, a importância dos fatores genéticos nos padrões de AF. Esta tarefa não tem sido fácil, uma vez que não é concensual o que se entende por padrão de $\mathrm{AF}$, tampouco, como se deve expressá-lo quantitativamente ${ }^{13}$. Assim, os principais objetivos desta pesquisa foram 1) descrever a noção de padrão de AF a partir de uma estatística designada de entropia aproximada (EA); 2) verificar a sua agregação fraterna em gêmeos de diferente zigotias e 3) estimar a contribuição dos fatores genéticos em tal medida do padrão de AF.

\section{PROCEDIMENTOS METODOLÓGICOS}

\section{Participantes}

Os dados utilizados neste estudo são oriundos dos participantes de "Encontros de Gêmeos" realizados em três Concelhos da Região Norte de Portugal (Viseu, Cantanhede e Maia). A organização dos eventos esteve a cargo da Faculdade de Desporto da Universidade do Porto e das respectivas Câmaras Municipais. A divulgação dos encontros foi realizada extensivamente via jornais, rádio e televisão. Também, foram enviadas "cartas convites" para todos os gêmeos devidamente cadastrados na "Associação Grupo Gêmeos de Portugal", a que se acrescentou divulgação pessoal entre as famílias cujos filhos são gêmeos.

O principal objetivo dos encontros era fornecer informações sobre a gemelaridade dos filhos aos seus pais. Secundariamente, enquanto as crianças e adolescentes estavam envolvidas em atividades lúdicas e recreativas, foram realizadas palestras sobre estilo de vida saudável para os pais, além de possibilitar a troca de experiência e convívio entre as famílias. Cada encontro conseguiu reunir, aproximadamente, 60 famílias.

O projeto foi aprovado pelo Concelho Científico da Faculdade de Desporto da Universidade do Porto e todas as famílias foram comunicadas sobre o objetivo do estudo e assinaram um termo de consentimento livre e esclarecido autorizando a participação voluntária dos seus filhos na pesquisa.

A amostra ficou constituída, exclusivamente, por 162 pares de gêmeos com idade entre seis e 18 anos (Tabela 1). 
Tabela 1. Distribuição dos pares de gêmeos da amostra por zigotia, sexo e idade.

\begin{tabular}{|c|c|c|c|c|}
\hline \multirow{2}{*}{ Zigotia } & \multirow{2}{*}{ Sexo } & \multirow{2}{*}{$\begin{array}{c}\text { Pares } \\
\text { (n) }\end{array}$} & \multicolumn{2}{|c|}{ Idade } \\
\hline & & & $m \pm d p$ & amplitude \\
\hline \multicolumn{5}{|l|}{$M Z$} \\
\hline & రం" & 25 & $12,6 \pm 0,6$ & $6-17$ \\
\hline & $Q$ & 52 & $13,1 \pm 0,5$ & $6-18$ \\
\hline \multicolumn{5}{|l|}{ DZ } \\
\hline & రం"ం & 22 & $13,4 \pm 0,4$ & $8-16$ \\
\hline & १९ & 35 & $13,2 \pm 0,5$ & $7-18$ \\
\hline \multicolumn{5}{|l|}{ DZso } \\
\hline & ợ & 28 & $12,4 \pm 0,5$ & $6-17$ \\
\hline
\end{tabular}

MZ: monozigóticos, DZ: dizigóticos, DZso: dizigóticos de sexo oposto

\section{Padrão de atividade física descrito pela entropia aproximada (EA)}

Steve Pincus, um matemático norte-americano, ao investigar problemas de regularidade e irregularidade de sinais biológicos, apresentou uma estatística sumária designada de $\mathrm{EA}^{14,15}$, cuja sensibilidade tem sido notável em pesquisas nos domínios de registros eletrocardiográficos ${ }^{16}$, alterações de foro psiquiátrico ${ }^{17,18}$, pulsões endocrinológicas ${ }^{19}$ e sinais fisiológicos ${ }^{15}$.

A EA examina a estrutura sequencial de um dado sinal (nesse caso a AF) e mede sua regularidade e complexidade no domínio do tempo. A sua formalização e justificação matemática podem ser consultadas em Pincus ${ }^{14}$. Ao contrário da média e desvio padrão que oferecem informações sobre a magnitude do sinal (variável), a EA oferece informações de como ele muda em função do tempo ${ }^{15}$. Esta medida utiliza um procedimento de probabilidade condicional para quantificar a estrutura sequencial ou regularidade com que o sinal evolui no tempo, determinando quanto ele pode exibir de padrões repetidos em sua "evolução" através do tempo. Em outras palavras, a sua padronização. A EA mede a verossimilhança com que um dado padrão, isto é, uma sequência de valores próximos para "n" observações, se mantém nas sequências seguintes dos registros. Maior verossimilhança de proximidade, isto é regularidade, produz menores valores de EA. Seus valores variam de zero a dois. Sinais altamente regulares e previsíveis, que contém uma ordem através do tempo, terão valores próximos a zero. Ao contrário, valores próximos a dois poderão ser constatados em sinais altamente irregulares, randômicos e imprevisíveis através do tempo ${ }^{14,15,20}$.

A Figura 1 apresenta uma das inovações do software Cine Wizard. Pode-se visualizar o "mapa trajetória" que descreve o padrão de valores da EA para o fenótipo AF, para cada membro do par de gêmeo, referente a cinco dias de avaliação.

Mapa Trajectória

IDGémeos: 188 Designação: Monozigótico Masculino Nomes: Luis Lopes; João Lopes

Idade: 10 Alturas: 150; 150 Pesos: 46;50 IMC's: 20,44; 22,22

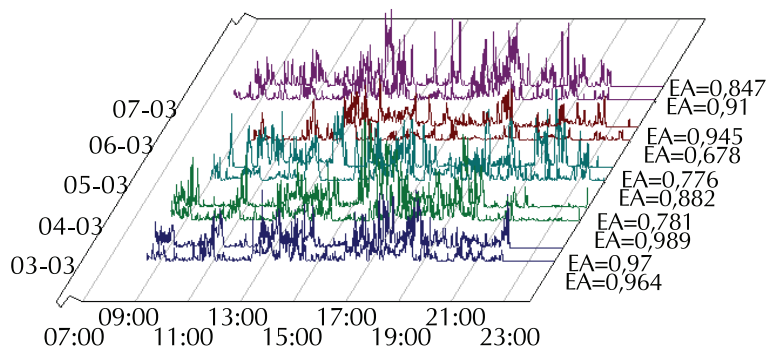

Figura 1. Mapa trajetória de registros de 5 dias de atividade física (AF) de um par de gêmeos. A linhas indicam o padrão de AF com estimativas da entropia aproximada (EA) para cada elemento do par.

\section{Software Cine Wizard}

Para descrever diversos modos de representação e quantificação do padrão de AF, foi desenvolvido, no Laboratório de Cineantropometria da Faculdade de Desporto, um software específico designado por Cine Wizard. Genericamente, este software tem implementado desde simples descrições numéricas de frequências de registros de níveis distintos de AF com base em valores de corte disponíveis para acelerômetros triaxiais ${ }^{21,22}$, até procedimentos mais complexos como a EA, registro "espectral sequencial" dos cinco dias de cada par, até a EA cruzada entre pares de gêmeos e análises de tendência a partir das séries de registros.

\section{Monitorização da atividade física (AF)}

Para a medida da AF, foi utilizado o acelerômetro Tritrac-R3D (medidas: 11,1 $66,7 \times 3,2 \mathrm{~cm}$, peso: 170 gr.). O Tritrac é um sensor de movimento tridimensional que tem a capacidade de medir o movimento em três planos (ântero-posterior, médio-lateral e vertical), capaz de avaliar a frequência, intensidade e duração dos movimentos referentes à $\mathrm{AF}$ realizada, posteriormente, sintetizando essa informação através do vetor de magnitude (Vmag). $\mathrm{O}$ Vmag é calculado como a raiz quadrada da soma do quadrado das acelerações para cada eixo, de acordo com a seguinte equação: $\sqrt{X^{2}+Y^{2}+Z^{2}}$.

$O$ dispêndio energético total diário é calculado somando a estimativa de taxa metabólica de repouso e o dispêndio energético em AF. A taxa 
metabólica de repouso é estimada tomando em consideração as variáveis idade, estatura, massa corporal e sexo. $\mathrm{O}$ dispêndio energético é então calculado convertendo o valor de Vmag através das equações fornecidas pelo fabricante.

O equipamento efetua registros de atividade em intervalos de tempo que variam de um segundo a um minuto, com capacidade de armazenamento da informação de 14 dias. $\mathrm{O}$ aparelho foi programado para realizar medições com intervalos de um minuto e entregue aos indivíduos após a introdução de informações relativas à estatura, à massa corporal, ao sexo e à idade. Os sujeitos foram instruídos a fixarem o aparelho na cintura e, para uma maior comodidade, o acelerômetro foi colocado em uma pequena bolsa para maior segurança.

A AF dos indivíduos foi monitorizada durante cinco dias de uma semana habitual. Foram avaliados três dias da semana (quinta, sexta e segunda-feira) e dois dias do final de semana (sábado e domingo). Para evitar variações sazonais, as avaliações decorreram durante os meses da primavera.

Todos os registros do acelerômetro foram convertidos para excel, onde apresentam informação relativa aos três planos, além dos valores do Vmag e valores de kcal derivados do Vmag. Através desse valor, foi possível converter a informação em METS, multiplicando os valores de kcal por $70 \mathrm{~kg}$, dividido pela massa corporal do indivíduo (em $\mathrm{kg}$ ) e novamente dividindo pelo tempo em minutos: $\left[\left(\mathrm{kcal}^{*} 70 /(\right.\right.$ massa corporal)/tempo em minutos].

\section{Determinação da gemelaridade}

A determinação da zigotia foi efetuada com recurso ao método direto de a nálise do DNA, que consiste em uma pequena coleta de sangue capilar o qual foi realizado por um indivíduo experiente, em cada membro do par de gêmeos.

A extração e amplificação do DNA por cadeia de reação da polimerase foi efetuada a partir das amostras de sangue obtidas dos 324 indivíduos através do método baseado na utilização da resina Chelex $^{23}$. Em todas as amostras de DNA, foi efetuada a análise autossômica de 17 STRs (CSF1PO, D2S1338, D3S1358, D5S818, D7S820, D8S1179, D13S317, D16S539, D18S51, D19S433, D21S11, FGA, PD, PE, TH01, TPO e VWA) e o locus da Amelogenina (determinação do sexo) através da amplificação por PCR, utilizando os kits comerciais Powerplex 16 System (Promega Corporation) e Identifiler (AB Applied Biosystems), de acordo com as instruções do fabricante.

A genotipagem foi realizada em aparelhos ABI
310 Genetic Analyzer (AB Applied Biosystems), de acordo com as instruções do fabricante, por determinação do tamanho dos fragmentos de DNA e comparação com escalas alélicas fornecidas com os kits comerciais. Nos casos em que os indivíduos apresentavam perfis genéticos de STRs idênticos, foi efetuado o cálculo de probabilidade de monozigotia, que segue, fundamentalmente, a metodologia de Essen-Møller ${ }^{24}$ e que se baseia nas frequências gênicas, neste caso, determinadas na população residente no Centro e Norte de Portugal.

\section{Procedimentos estatísticos}

A análise estatística foi realizada em diferentes etapas com graus de complexidade distintas ao longo do estudo. Os programas estatísticos utilizados foram o SYSTAT 10.0, STATA 10,0 e o Twinan92. A EA foi calculada para três diferentes fenótipos de AF, classificados de acordo com a quantidade de dias de mensuração. Foram consideradas a EA da AF avaliada durante os cinco dias (EA 5 dias), três dias da semana (EA 3 dias) e dois dias do final de semana (EA 2 dias). Em uma primeira etapa foi realizada a análise exploratória para verificar possíveis erros na entrada de informações, a presença de outliers e a normalidade das distribuições, bem como calcular a média, desvio padrão e amplitude de variação. Também, utilizou-se o recurso a modelos distintos para melhor descrever o padrão das matrizes de covariância entre pares de gêmeos (variância genética, envolvimento comum e único, $\left.\mathrm{a}^{2}, \mathrm{c}^{2}, \mathrm{e}^{2}\right)$. O nível de significância foi mantido em 5\%.

\section{RESULTADOS}

A análise exploratória dos dados não apresentou violação da normalidade e valores extremos; os valores da assimetria e do achatamento são relativamente baixos (Tabela 2).

Posteriormente, e face à presença de enorme variação nos valores das idades dos gêmeos, foi realizada uma análise de regressão dos três fenótipos do padrão de AF (EA 5, 3 e 2 dias) com as covariáveis idade, sexo, idade*sexo, idade ${ }^{2}$, idade $^{2 *}$ sexo. Nenhum destes preditores apresentou qualquer significado estatístico ( $\mathrm{p}>0,05)$, implicando um $\mathrm{R}^{2}=0$. Assim, não houve necessidade de calcular resíduos da regressão.

A Figura 2 apresenta os diagramas de dispersão dos gêmeos MZ e DZ. Através da análise visual pode-se perceber a maior correlação intra-par nos valores da EA para os três fenótipos da AF para os gêmeos MZ. Essa maior correlação representa uma 
Tabela 2. Medidas descritivas do fenótipo EA 5 dias dos gêmeos de acordo com a zigotia.

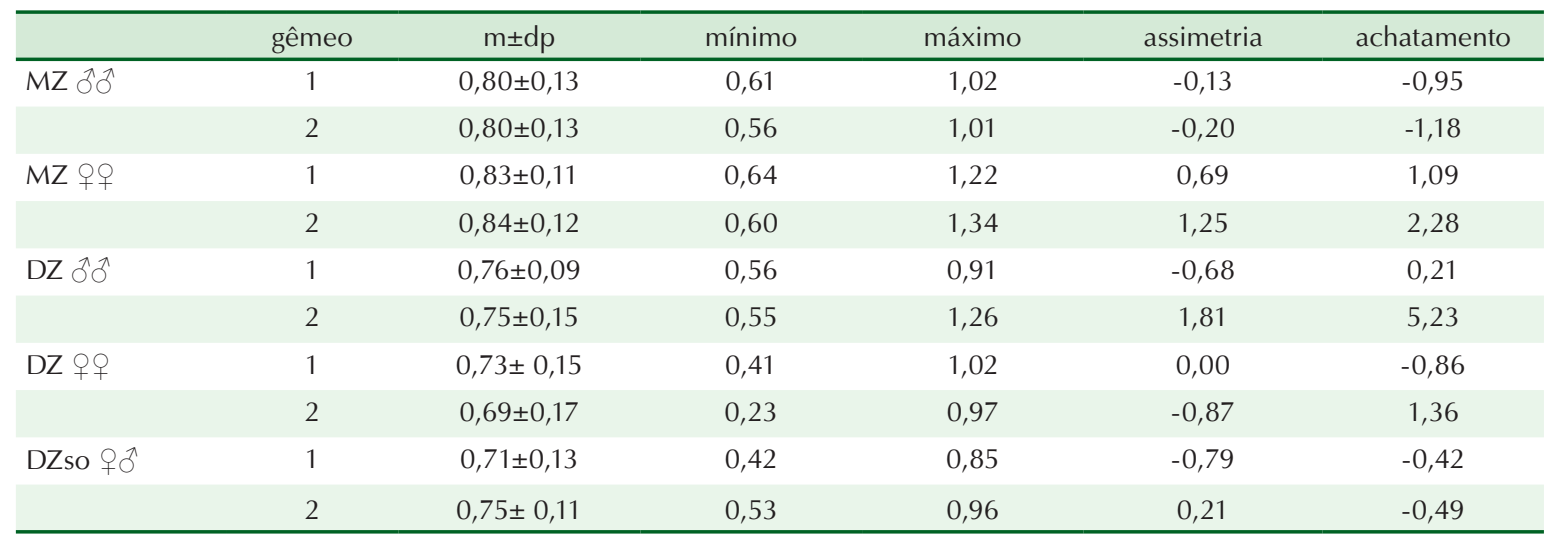

MZ: gêmeos monozigóticos, DZ: gêmeos dizigóticos, DZso: gêmeos dizigóticos de sexo oposto, m: média, dp: desvio padrão.
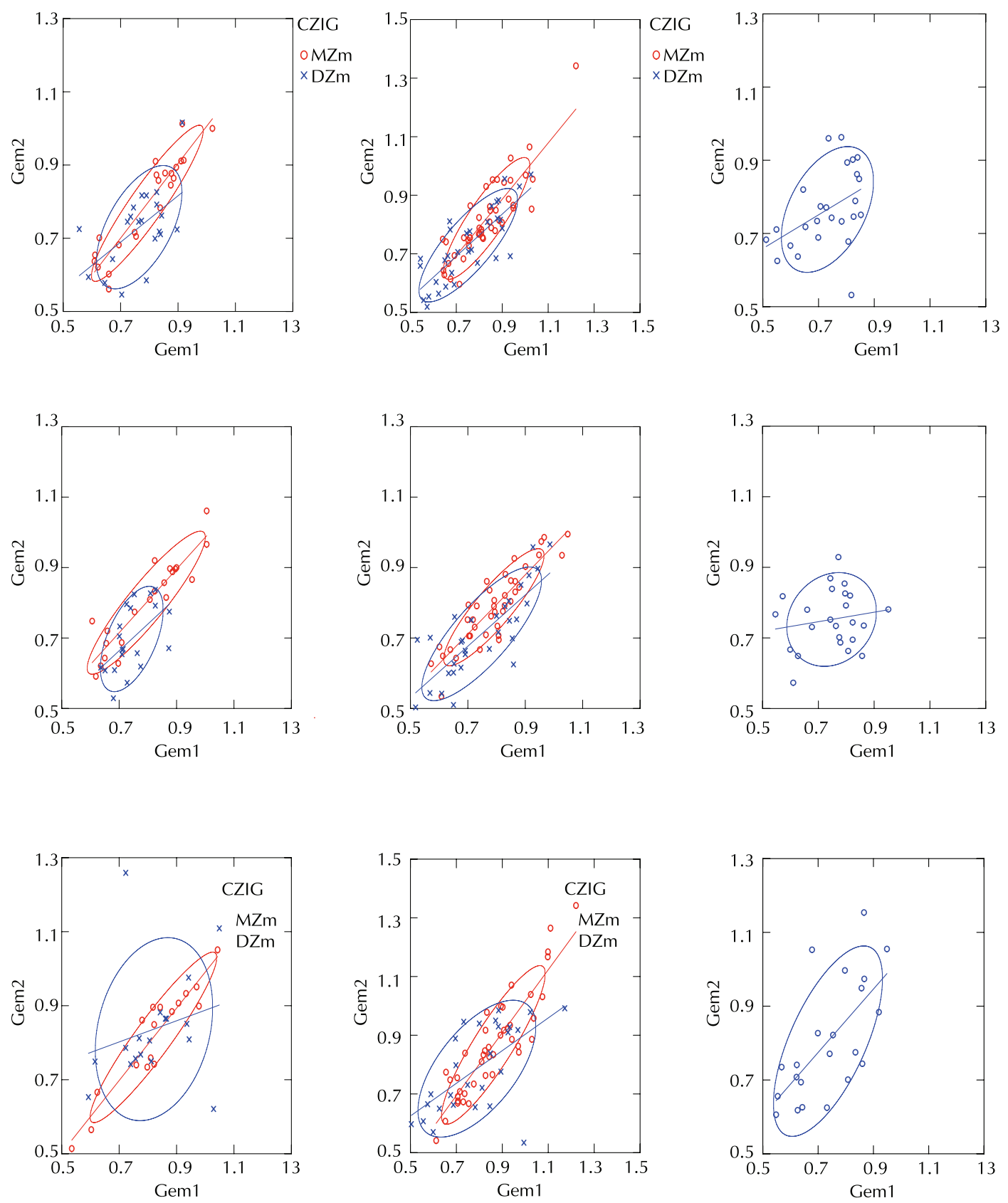

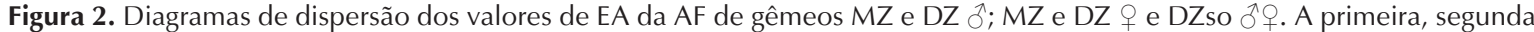
e terceira linha de gráficos refere-se aos fenótipos EA 5, 3 e 2 dias, respectivamente. 
maior homogeneidade e, consequentemente, uma elipse mais compacta.

O coeficiente de correlação intraclasse (CCI) é uma estatística frequentemente utilizada para sumarizar o grau de homogeneidade de uma classe de valores; espera-se que a classe dos gêmeos $\mathrm{MZ}$ para qualquer traço fenotípico seja sempre mais homogênea que a classe dos gêmeos DZ. Nesse sentido, os valores de correlação intraclasse do gêmeos monozigóticos $\left(t_{\mathrm{MZ}}\right)$ devem ser sempre superiores aos de gêmeos DZ (Tabela 3). Os gêmeos $\mathrm{MZ}$ apresentaram valores de correlação intraclasse superiores aos gêmeos DZ nos três fenótipos estudados.

Tabela 3. Coeficientes de correlação intraclasse $(\mathrm{CCl})$ e respectivos valores de prova para os diferentes fenótipos da EA.

\begin{tabular}{lcccc}
\hline \multirow{2}{*}{ Fenótipos } & \multicolumn{2}{c}{ Gêmeos MZ } & \multicolumn{2}{c}{ Gêmeos DZ } \\
\cline { 2 - 5 } & $\mathrm{CCl}$ & $\mathrm{p}$ & $\mathrm{CCl}$ & $\mathrm{p}$ \\
\hline EA 5 dias & 0,89 & $<0,001$ & 0,68 & $<0,001$ \\
EA 3 dias & 0,87 & $<0,001$ & 0,59 & $<0,001$ \\
EA 2 dias & 0,89 & $<0,001$ & 0,65 & $<0,001$ \\
\hline
\end{tabular}

p: valor de prova

O recurso a modelos distintos para melhor descrever o padrão das matrizes de covariância entre pares de gêmeos ${ }^{25}$, mostrou que o efeito do ambiente único $\left(\mathrm{e}^{2}\right)$ se apresenta como um preditor de pouca influência para a explicação das diferenças interindividuais nos três fenótipos. A proporção de variação relacionada com fatores do ambiente comum $\left(\mathrm{c}^{2}\right)$ assume uma importância considerável, com cerca de $45 \%$ da variação total no fenótipo EA 5 dias. Presente nos três fenótipos, embora com menor magnitude no fenótipo EA 5 dias (44\%), é de se considerar que a maior parcela de importância deve ser atribuída aos fatores genéticos, fato assim demonstrado pelos elevados valores apresentados nos fenótipos EA 3 e 2 dias (89\%).

Tabela 4. Estimativa dos parâmetros ( \pm erro-padrão) dos melhores modelos para descrever os efeitos genéticos $\left(\mathrm{a}^{2}\right)$, do ambiente comum $\left(\mathrm{c}^{2}\right)$ e único $\left(\mathrm{e}^{2}\right)$ dos diferentes fenótipos da EA.

\begin{tabular}{lcccc}
\hline Fenótipo & Modelo & $\mathbf{a}^{2}$ & $\mathbf{c}^{2}$ & $\mathbf{e}^{2}$ \\
\hline EA 5 dias & ACE & $0,44 \pm 0,11$ & $0,45 \pm 0,11$ & $0,11 \pm 0,02$ \\
EA 3 dias & AE & $0,89 \pm 0,02$ & - & $0,11 \pm 0,02$ \\
EA 2 dias & AE & $0,89 \pm 0,02$ & - & $0,11 \pm 0,02$ \\
\hline
\end{tabular}

\section{DISCUSSÃO}

Um dos objetivos deste estudo foi descrever a noção de padrão de AF a partir de uma estatística desig- nada de entropia aproximada (EA). Entretanto, a expressão padrão de AF vem sendo utilizada com frequência na literatura apesar de sua compreensão e operacionalização serem ainda inconsistentes ${ }^{13}$. Por apresentar características extremamente complexas e únicas, é de se compreender que a sua operacionalização e interpretação sejam também complexas ${ }^{4-6}$. A EA fornece uma nova forma de analisar as respostas da AF provenientes de um acelerômetro para caracterizar seus padrões. Devido ao fato da AF ser um fenômeno complexo e multifatorial, muito caracterizada na infância por atividades extremamente esporádicas, de forte intermitência com pulsos de intensidade e duração distintos, a EA parece ser uma medida interessante no intuito de suprir a inexistência de estatísticas "simples" com o objetivo de descrever seu padrão. Esta estatística foi desenvolvida para quantificar a regularidade de séries de dados, inclusive, se forem de curta duração e extremamente irregulares. A EA assinala um valor nulo ou positivo à série temporal; valores maiores indicam maior complexidade e irregularidade do sina ${ }^{20}$. Ainda que este seja um sinal extremamente complexo, a aplicação da EA no auxílio do entendimento do padrão de AF encontra-se em fase inicial, sendo a sua aplicação pioneira na presente pesquisa.

É importante salientar que o estudo do padrão de AF pode fornecer informações preciosas no sentido de entender como são organizadas as atividades diárias de crianças e/ou adolescentes. Porém, sabemos que grande parte das atividades diárias infantis é governada muito em função do horário escolar ${ }^{26}$. Assim, espera-se alguma regularidade nos comportamentos.

As recomendações atuais de AF dão conta de uma periodicidade de 60 min diários, com intensidade moderada a elevada, para que, desta forma, proporcionem benefícios à saúde ${ }^{1}$. Contudo, a informação disponível preocupa-se em entender quanto de AF é exercida por crianças e adolescentes ao longo dos dias da semana. Sua operacionalização, atualmente, é efetuada à partir dos níveis de $\mathrm{AF}$, não se preocupando com os seus padrões. Nesse sentido, o estudo do padrão de AF com o auxílio da EA, traznos dados mais consitentes, possibilitando observar as atividades realizadas ao longo do dia, minuto a minuto, o que consiste em informação substancial para compreendermos a distribuição da AF e como se caracteriza em termos do seu padrão.

Não conseguimos localizar informação empírica sobre variação nos padrões de AF em delineamentos com sujeitos aparentados. Com base no delineamento gemelar, a informação produzida 
neste estudo salienta uma forte contribuição genética, entre 44 e $89 \%$ da variação total nos três fenótipos avaliados.

Em um estudo inovador acerca da influência dos fatores genéticos e ambientais na AF, Duncan et al. ${ }^{27}$ efetuaram a medida da AF através de inquérito desenvolvido pela University of Washington Twin Registry (UWTR), em uma amostra de 1.389 pares de gêmeos adultos com idades compreendidas entre 29 e 33 anos. Este registro procurou quantificar quantas vezes por semana os sujeitos exerciam AF moderada e vigorosa por, pelo menos, 30 e 20 minutos, respectivamente. Os autores criaram duas variáveis distintas com base em diferentes pontos de corte do total de AF: 1) $\geq 60 \mathrm{~min} / \mathrm{sem}$, conforme o que é utilizado no estudo GenomEUtwin e 2) $\geq 150 \mathrm{~min} / \mathrm{sem}$, seguindo as recomendações do ACSM/CDC. Foi verificado que para o ponto de corte $\geq 60 \mathrm{~min} / \mathrm{sem}, \mathrm{r}_{\mathrm{MZ}}=0,43(0,33-0,54)$ e $\mathrm{r}_{\mathrm{Dz}}=0,30$ $(0,12-0,47)$. Estas correlações foram atenuadas quando utilizaram o ponto de corte $\geq 150 \mathrm{~min} / \mathrm{sem}$ : $r_{M Z}=0,30(0,20-0,40)$ e $r_{D z}=0,25(0,07-0,42)$. Estes resultados sugerem que os fatores do ambiente comumente partilhado têm forte influência na AF.

Stubbe et al. ${ }^{9}$, em um estudo com 2.628 pares de gêmeos (443 MZð̄, 652 MZㅇ, 434 DZㅇ e 722 DZso $\widehat{\jmath}$ 우), com idades entre 13 e 20 anos, apresentaram valores totalmente contrários aos anteriormente citados. Os autores referem que um conjunto principal de efeito da idade e sexo foi encontrado sobre a participação desportiva. Pares de gêmeos mais novos apresentaram maior participação desportiva (13-14 anos, $\mathrm{r}=0,47-0,88 ; 15-16$ anos, $\mathrm{r}=0,46-0.83$ ) que gêmeos mais velhos (17-18 anos $\mathrm{r}=0,18-0,88$; 19-20 anos, $r=0,35-0,86$ ). Para cada faixa etária, os meninos apresentaram valores superiores. As análises de semelhança genética mostraram uma mudança nos fatores que contribuem para a participação desportiva desde a adolescência até à idade adulta. Entre as idades de 13-14 anos os valores foram: $c^{2}=84 \%$ e $e^{2}=16 \% ; 15-16$ anos: $c^{2}=78 \%$ e $\mathrm{e}^{2}=22 \% ; 17-18$ anos: $\mathrm{a}^{2}=36 \%, \mathrm{c}^{2}=47 \%$ e e $\mathrm{e}^{2}=17 \% ; 19$ 20 anos: $\mathrm{a}^{2}=85 \%$ e e $\mathrm{e}^{2}=15 \%$.

No presente estudo, a EA ressalta a forte presença de influência dos fatores genéticos. Contudo, devemos salientar a ausência de pesquisa com metologia semelhante. Ainda assim, os resultados aqui encontrados podem ser suportados por outras pesquisas. Maia et al..$^{28}$ estudaram dois fenótipos distintos, mas que refletem os níveis de AF: índice de participação desportiva e tempo de AF de lazer. O fracionamento da variancão total foi o seguinte: índide de participação esportiva no sexo masculino: $\mathrm{a}^{2}=68,4 \%, \mathrm{c}^{2}=20 \%$ e e $\mathrm{e}^{2}=11,6 \%$ e no sexo feminino: $\mathrm{a}^{2}=39,8 \%, \mathrm{c}^{2}=28,4 \%$ e $\mathrm{e}^{2}=31,8 \%$. Na AF de lazer dos meninos, os fatores genéticos explicaram $63 \%$ da variação total, o ambiente único explicou $37 \%$ e o ambiente comum não apresentou valores significativos. No sexo feminino, os resultados foram $a^{2}=32 \%, c^{2}=38 \%$ e $e^{2}=30 \%$.

Contrapondo o estudo supracitado, encontramos o trabalho desenvolvido por Franks et al. ${ }^{29}$, em que a variância para o fenótipo dispêndio energético foi fracionado do seguinte modo: $\mathrm{a}^{2}=19 \%$, $\mathrm{c}^{2}=59 \%$ e $\mathrm{e}^{2}=23 \%$. A taxa metabólica de repouso apresentou valores de $\mathrm{c}^{2}=65 \%$ e e $\mathrm{e}^{2}=35 \%$. Estes resultados sugerem que as semelhanças familiares na AF podem ser explicadas predominantemente pelo envolvimento partilhado e não pela variabilidade genética. $O$ fenótipo dispêndio energético em AF apresentou valores que ressaltam sobremaneira a importância dos fatores ambientais, onde $\mathrm{a}^{2}=0 \%$, $\mathrm{c}^{2}=69 \%$ e e $\mathrm{e}^{2}=31 \%$. Eriksson et al. ${ }^{30}$, verificaram que os fatores genéticos foram de $49 \%$ e a variância residual de $51 \%$.

Um aspecto importante a considerar nesta pesquisa refere-se ao uso de procedimentos de análise pouco convencionais na área. Ainda que tenha sido realizado um esforço interpretativo dos padrões de AF com a utilização da EA, devemos ter algum cuidado na extrapolação dos resultados aqui encontrados, uma vez que se referem, exclusivamente, a gêmeos. Contudo, nada nos diz que sujeitos não aparentados tenham padrões de AF completamente distintos, face à circunstância de uma parte substancial das rotinas de vida de crianças e jovens ser governada pelos horários escolares. A falta de estudos empíricos com metodologia semelhante, limita a possibilidade de comparação com os resultados de outras pesquisas. Não obstante estas limitações, a que acrescentamos o fato da amostra ser de dimensão reduzida, não ter sido possível dividí-la em diferentes grupos etários e explorar toda a potencialidade dos cinco grupos gemelares, pensamos que a EA apresenta uma nova possibilidade de pesquisa que auxiliará para a melhor compreensão do comportamento em AF independente do seu modo, intensidade e duração. Sugere-se que futuros estudos devam ser conduzidos em amostras com indivíduos jovens e adultos, aparentados ou não, com o intuito de verificar a consistência dos resultados aqui encontrados. Importante seria associar os padrões de AF a fatores de risco metabólico, obesidade e outras características menos saudáveis para se perceber a sua potencialidade interpretativa e de intervenção. 


\section{CONCLUSÃO}

Com base nos resultados pode-se concluir que 1) a EA é uma medida possível para descrever o padrão de AF que apresenta informações consistentes, as quais possibilitam a descrição das atividades realizadas ao longo do dia; 2) foi verificado uma maior homogeneidade na EA dos três fenótipos da AF entre os gêmeos MZ, demonstrando, assim, a presença de agregação fraterna e 3) os fatores genéticos apresentam forte influência na variação interindividual no padrão de AF descrita pela EA e sua contribuição variou entre 44 e $89 \%$. Também ficou evidente a importância dos fatores ambientais, especialmente, os comumente partilhados (45\%).

Existe a necessidade de atribuir significado à interpretação nos níveis de AF de crianças e jovens. A variabilidade nos seus padrões é bastante complexa o que exige uma forma plural de o entender. Este estudo pioneiro acerca da metodologia, necessita novas extensões para melhor compreensão da sua importância em termos epidemiológicos e de intervenção na AF, saúde e bem-estar de crianças e jovens.

\section{REFERÊNCIAS BIBLIOGRÁFICAS}

1. Strong WB, Malina RM, Blimkie CJ, Daniels SR, Dishman RK, Gutin B, et al. Evidence based physical activity for school-age youth. J Pediatr 2005;146:732-7.

2. Bauman AE. Updating the evidence that physical activity is good for health: an epidemiological review 2000-2003. J Sci Med Sport 2004;7:6-19.

3. Twisk JW. Physical activity guidelines for children and adolescents: a critical review. Sports Med 2001;3:617-27.

4. Van Der Horst K, Paw MJ, Twisk JW, Van Mechelen W. A brief review on correlates of physical activity and sedentariness in youth. Med Sci Sports Exerc 2007;39:1241-50.

5. Hinkley T, Crawford D, Salmon J, Okely AD, Hesketh K. Preschool children and physical activity: a review of correlates. Am J Prev Med 2008;34:435-41.

6. Sallis JF, Prochaska JJ, Taylor WC. A review of correlates of physical activity of children and adolescents. Med Sci Sports Exerc 2000;32:963-975.

7. Seabra AF, Mendonca DM, Goring HH, Thomis MA, Maia JA. Genetic and environmental factors in familial clustering in physical activity. Eur J Epidemiol 2008;23:205-11.

8. Mattocks C, Ness A, Deere K, Tilling K, Leary S, Blair $\mathrm{SN}$, et al. Early life determinants of physical activity in 11 to 12 year olds: cohort study. BMJ 2008;336:26-9.

9. Stubbe JH, Boomsma DI, Vink JM, Cornes BK, Martin NG, Skytthe A, et al. Genetic influences on exercise participation in 37,051 twin pairs from seven countries. PLoS ONE 2006;1:e22.
10. Beunen G, Thomis M. Genetic determinants of sports participation and daily physical activity. Int J Obes Relat Metab Disord 1999;23:S55-63.

11. Maia JAR, Lopes VP, Seabra A, Garganta R. Efeitos genéticos e do envolvimento nos níveis de actividade física e aptidão física associada à saúde. Um estudo em gémeos dos 6 aos 12 anos de idade do Arquipélago dos Açores (Portugal). Rev Bras Ciên Mov 2003;11:37-44.

12. Carlsson S, Andersson T, Lichtenstein P, Michaelsson K, Ahlbom A. Genetic effects on physical activity: results from the Swedish Twin Registry. Med Sci Sports Exerc 2006;38:1396-401.

13. Lima RBCP, Fermino RC, Seabra A, Garganta R, Maia JAR. Padrão de atividade física em crianças e jovens: Um breve resumo do estado do conhecimento. Rev Bras Cineantropom Desempenho Humano. 2010;12:68-76.

14. Pincus SM. Approximate entropy as a measure of system complexity. Proc Natl Acad Sci USA 1991;88:2297-301.

15. Pincus SM, Goldberger AL. Physiological time-series analysis: what does regularity quantify? Am J Physiol 1994;266:H1643-56.

16. Schuckers SAC. Use of approximate entropy measurements to classify ventricular tachycardia and fibrillation. J Electrocardiol 1998;31:101-5.

17. Caldirola D, Bellodi L, Caumo A, Migliarese G, Perna G. Approximate entropy of respiratory patterns in panic disorder. Am J Psychiatry 2004;161:79-87.

18. Pincus SM. Approximate entropy as a measure of irregularity for psychiatric serial metrics. Bipolar Disord 2006;8:430-40.

19. Pincus SM, Veldhuis JD, Rogol AD. Longitudinal changes in growth hormone secretory process irregularity assessed transpubertally in healthy boys. Am J Physiol Endocrinol Metab 2000;279:E417-24.

20. Pincus SM. Assessing serial irregularity and its implications for health. Ann N Y Acad Sci 2001;954:245-67.

21. Rowlands AV, Thomas PW, Eston RG, Topping R. Validation of the RT3 triaxial accelerometer for the assessment of physical activity. Med Sci Sports Exerc 2004;36:518-24.

22. Freedson P, Pober D, Janz KF. Calibration of accelerometer output for children. Med Sci Sports Exerc 2005;37:S523-30.

23. Coombs NJ, Gough AC, Primrose JN. Optimisation of DNA and RNA extraction from archival formalin-fixed tissue. Nucleic Acids Res 1999;27:e12.

24. Essen-Møller E. Die beweiskraft der ahnlichkeit im vaterschftsnachweiss. Theoretische brundlagen. Mitt Anthrop Ges Wien 1939;68:9-53.

25. Liu PY, Pincus SM, Keenan DM, Roelfsema F, Veldhuis JD. Analysis of bidirectional pattern synchrony of concentration-secretion pairs: implementation in the human testicular and adrenal axes. Am J Physiol Regul Integr Comp Physiol 2005;288:R440-6.

26. Silva SP, Magalhães M, Garganta RM, Seabra AF, Bustamente A, Maia JAR. Padrão de actividade física de escolares. Rev Bras Cineantropom Desempenho Hum 2006;8:19-26. 
27. Duncan GE, Goldberg J, Noonan C, Moudon AV, Hurvitz P, Buchwald D. Unique environmental effects on physical activity participation: a twin study. PLoS ONE 2008;3:e2019.

28. Maia JA, Thomis M, Beunen G. Genetic factors in physical activity levels: a twin study. Am J Prev Med 2002;23:87-91.

29. Franks PW, Ravussin E, Hanson RL, Harper IT, Allison DB, Knowler WC, et al. Habitual physical activity in children: the role of genes and the environment. Am J Clin Nutr 2005;82:901-8.

30. Eriksson M, Rasmussen F, Tynelius P. Genetic factors in physical activity and the equal environment assumption - the Swedish young male twins study. Behav Genet 2006;36:238-47.

\section{Endereço para correspondência}

José António Ribeiro Maia

Laboratório de Cineantropometria e Gabinete de Estatística Aplicada

Universidade do Porto. Faculdade de Desporto Rua Plácido Costa, 91 4200-450, Porto. Portugal

E-mail: jmaia@fade.up.pt 